\title{
Benign Enhancing Foramen Magnum Lesions: Clinical Report of a Newly Recognized Entity
}

\author{
(D)B.J. McGuinness, DJ.P. Morrison, (D) S.K. Brew, and (D) M.W. Moriarty
}

\begin{abstract}
SUMMARY: Intradural extramedullary foramen magnum enhancing lesions may be due to meningioma, nerve sheath tumor, aneurysm, or meningeal disease. In this clinical report of 14 patients, we describe a novel imaging finding within the foramen magnum that simulates disease. The lesion is hyperintense on 3D-FLAIR and enhances on 3D gradient-echo sequences but is not seen on 2D-TSE T2WI. It occurs at a characteristic location related to the posterior aspect of the intradural vertebral artery just distal to the dural penetration. Stability of this lesion was demonstrated in those patients who underwent follow-up imaging. Recognition of this apparently benign lesion may prevent unnecessary patient anxiety and repeat imaging.
\end{abstract}

f

ntradural extramedullary enhancing lesions in the region of the foramen magnum have a differential diagnosis of meningioma, schwannoma, aneurysm, or meningeal manifestation of malignant or inflammatory disease. Accurate imaging diagnosis of such lesions, even if benign, is important because lesion growth in such a confined space can have severe clinical sequelae through mass effect on the lower medulla and upper cervical cord. Surgical resection within the foramen magnum has major risks and challenges despite advancements in surgical approaches. ${ }^{1,2}$

At our institution, we have recognized an enhancing lesion that occurs at a characteristic location within the foramen magnum in a series of 14 patients. Here, we discuss the location and imaging characteristics of an apparently benign and nonprogressive lesion.

\section{CASE SERIES}

\section{Case Selection}

Local regional ethics approval was obtained for this retrospective study. Fourteen patients with small, enhancing foramen magnum lesions were identified by MR imaging during a 5-year period between May 2011 and May 2016. The lesions had been identified

\footnotetext{
Received September 22, 2016; accepted after revision December 3.
}

From Trinity MRI (B.J.M., J.P.M., S.K.B., M.W.M.), Auckland, New Zealand; and Neuroradiology Section (B.J.M., S.K.B., M.W.M.), Department of Radiology, Auckland City Hospital, Auckland, New Zealand.

Please address correspondence to Ben McGuinness, MD, Trinity MRI, 96C Carlton Gore Rd, Newmarket, Auckland 1023, New Zealand; e-mail: benmcg@gmail.com

三 Indicates article with supplemental on-line table.

http://dx.doi.org/10.3174/ajnr.A5085 during the initial reporting and double-read by 2 fellowshiptrained neuroradiologists as per the routine practice of the study institution (Trinity MRI, Auckland, New Zealand). Lesion size, location, and signal intensity on the available sequences performed were subsequently recorded by one of the neuroradiologists (B.J.M.). Patient demographic data, indication for scanning, relevant medical history, and latest follow-up were retrospectively obtained by review of the PACS data base of the imaging center.

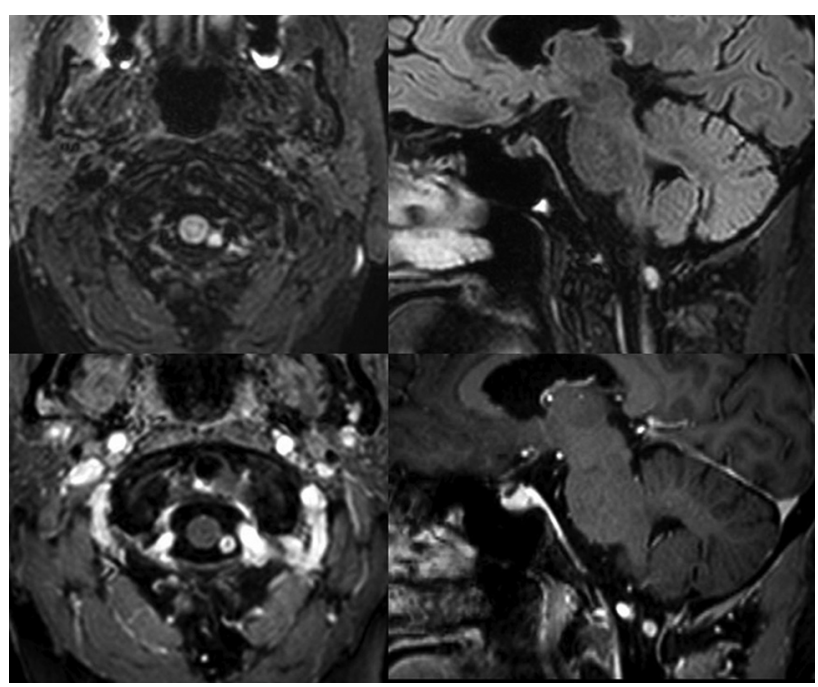

FIG 1. Axial (upper left) and sagittal (upper right) reformats of a 3DFLAIR sequence show a 6-mm hyperintense lesion within the left posterior aspect of the foramen magnum. This lesion shows enhancement on the axial (lower left) and sagittal (lower right) reformats of the 3D-T1-weighted postcontrast sequences. The lesion lies posteromedial to the dural penetration of the left vertebral artery. 


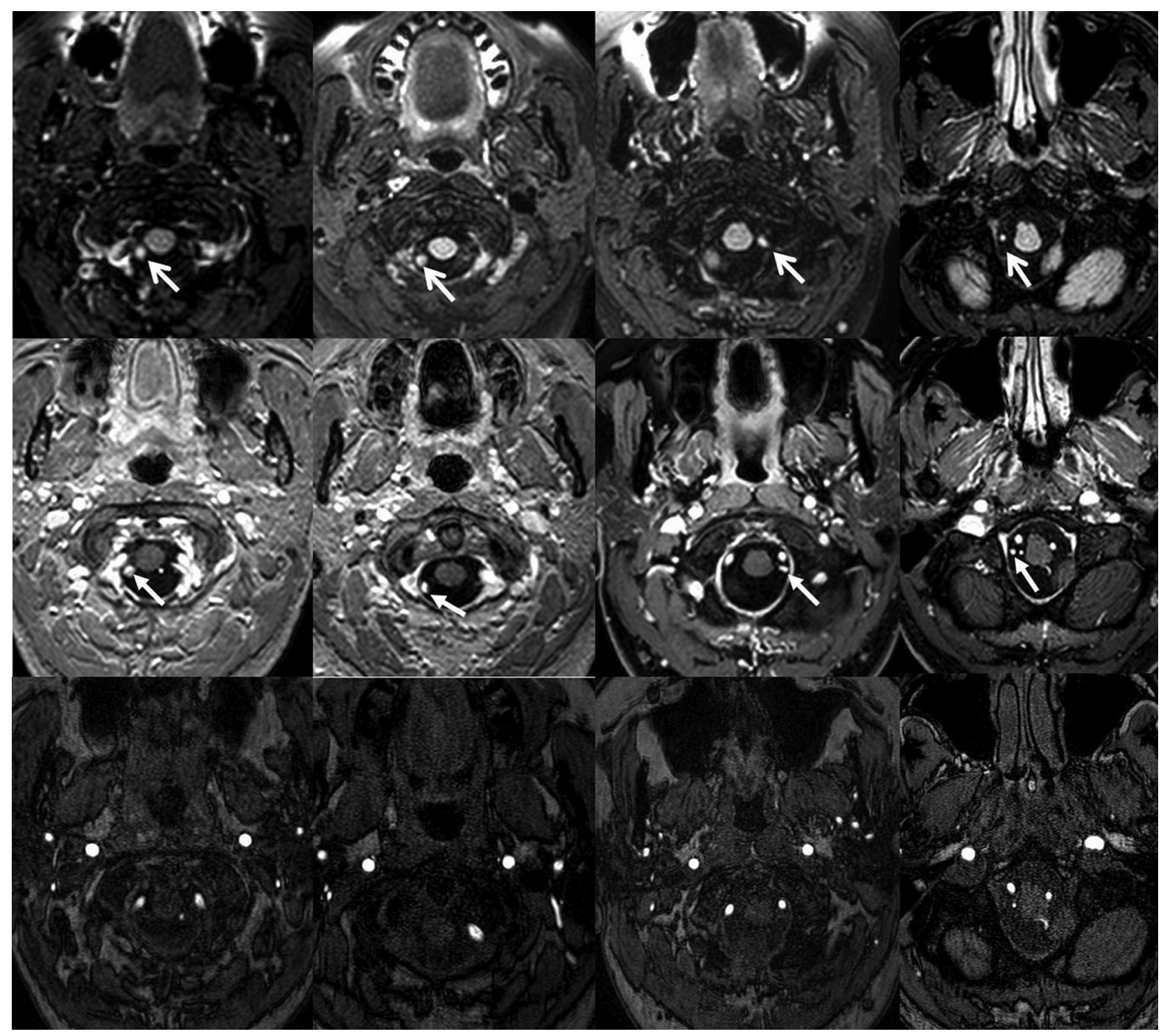

FIG 2. Four different examples of foramen magnum lesions. 3D-FLAIR images (upper row) show lesions as hyperintense foci near the posterior aspect of the dural penetration of the vertebral artery (white arrows). Corresponding 3D-T1-weighted postcontrast images (center row) show that the lesions enhance (white arrows). TOF-MRA axial source images (lower row) demonstrate the relationship of local arteries to the lesions, which do not show flow-related signal return.

Comparison with any available prior or subsequent imaging was also performed to ascertain maximal longitudinal stability of the lesions.

\section{Imaging Acquisition}

All MR imaging was performed on a 3T Achieva scanner (Philips Healthcare, Best, the Netherlands). We performed the following precontrast sequences: sagittal T1WI inversion recovery TSE, axial and coronal T2WI TSE, sagittal 3D-FLAIR TSE $(\mathrm{TR}=4800$ $\mathrm{ms}, \mathrm{TE}=250-284 \mathrm{~ms}, \mathrm{TI}=1650 \mathrm{~ms}$, matrix $=192 \times 190$, voxel size $=0.6 \times 0.6, \mathrm{FOV}=230 \times 230,1.2$-mm-thick, flip angle $=$ $90^{\circ}$, TSE factor $=182$ ), axial DWI, and axial SWI. Following the administration of $7.5 \mathrm{~mL}$ of gadobutrol (Gadovist, $1 \mathrm{mmol} / \mathrm{mL}$; Bayer Schering Pharma, Berlin, Germany), an axial 3D-T1weighted inversion recovery-prepped turbo field echo was performed with or without fat saturation $(\mathrm{TR}=5.6-8 \mathrm{~ms}$, $\mathrm{TE}=$ 2.7-4 ms, T1 $=1000 \mathrm{~ms}$, matrix $=240 \times 202$, voxel size $=1 \times 1$,
FOV $=240 \times 200,1$-mm-thick, flip angle $=8$, turbo field echo $=$ 230). In 2 cases, an axial T2 driven equilibrium radiofrequency reset pulse TSE imaging was performed through the lesion before contrast administration $(\mathrm{TR}=1500 \mathrm{~ms}, \mathrm{TE}=203 \mathrm{~ms}$, matrix $=$ $312 \times 312$, voxel size $=0.48 \times 0.48, \mathrm{FOV}=150 \times 150,0.48-\mathrm{mm}-$ thick, flip angle $=90^{\circ}$, TSE factor $=41$ ).

\section{RESULTS}

Sixteen cases of small, enhancing foramen magnum lesions were identified in 14 patients. The patients ranged from 26 to 78 years of age, with 8 men and 6 women. The lesions ranged from 2 to $6 \mathrm{~mm}$ and always lay closely related to the posterior aspect of the vertebral artery in the subarachnoid space just distal to the dural penetration of this artery. Fourteen lesions were separated from the posterior intradural vertebral artery by a cleft of CSF measuring $\leq 2 \mathrm{~mm}$. Two lesions were separated from the artery by 3 and $5 \mathrm{~mm}$. The center of the 


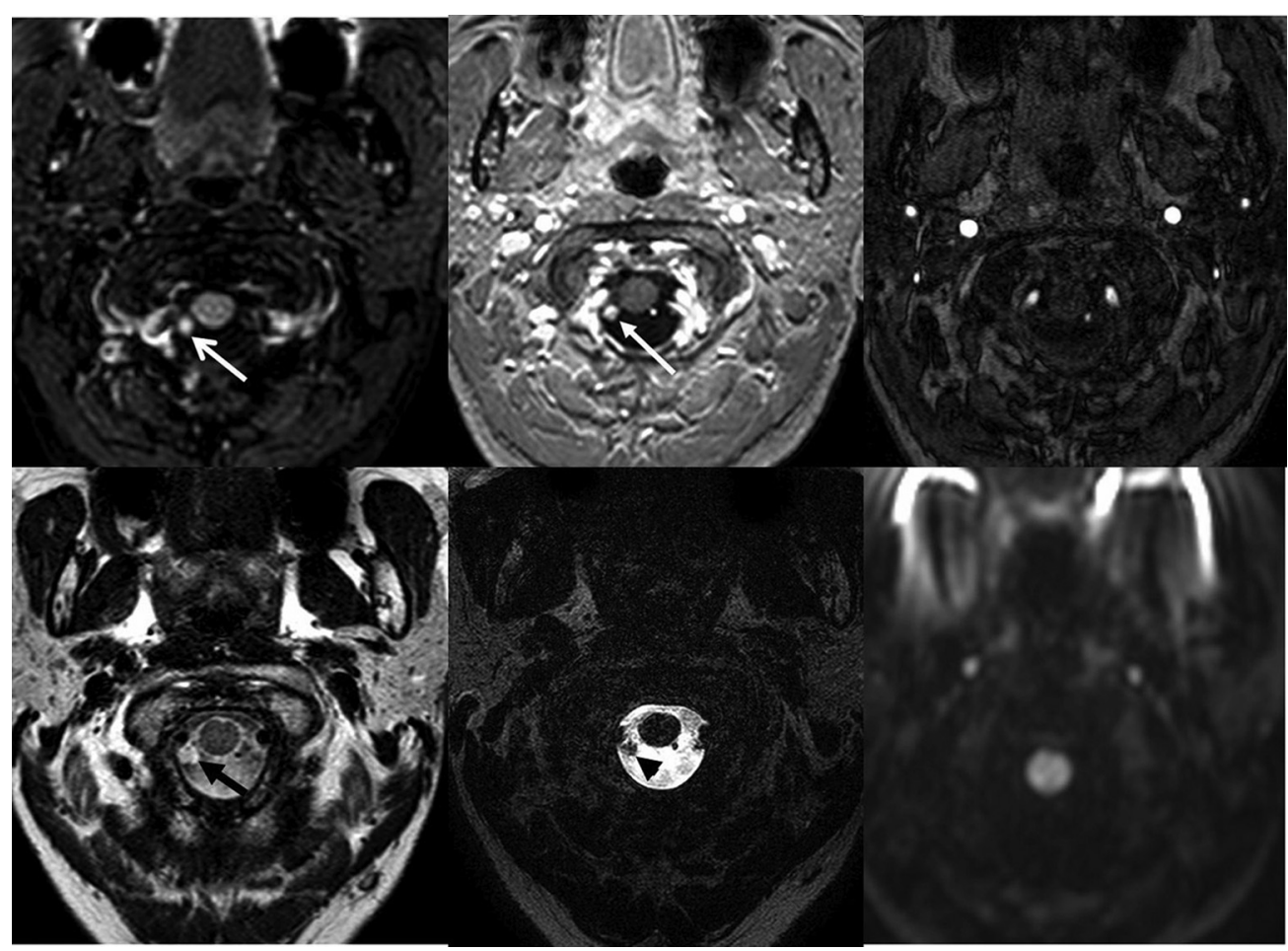

FIG 3. Axial reformats of a 3D-FLAIR (upper left image) sequence show a 4-mm hyperintense focus within the right side of the foramen magnum just posterior to the dural penetration of the right vertebral artery (white arrow). Axial reformat of a 3D-T7-weighted postcontrast sequence (upper center image) shows that this lesion enhances (white arrow). The lesion is isointense with CSF on a TSE T2-weighted (lower left image, black arrow) and hypointense on a TSE T2-weighted image with a $90^{\circ}$ flip-back pulse (T2 driven equilibrium radiofrequency reset pulse, lower center image, black arrowhead). The lesion is occult on TOF-MRA (upper right image) and B1000 (lower right image).

lesion was a median of $6 \mathrm{~mm}$ below the foramen magnum (range, 0-10 mm). Seven patients had lesions that were right-sided; 5, leftsided; and 2, bilateral. No lesion contacted the posterior inferior cerebellar artery regardless of the level of the origin of this artery from the intradural vertebral artery. No lesion contacted the dura or the spinal cord. All lesions lay below the rootlets of the ninth, tenth, and twelfth cranial nerves. The rootlets of the spinal accessory nerve and the first cervical spinal nerve were not able to be determined in relation to the lesions.

The lesions were round and ovoid and shared the same signal intensity on different imaging sequences (Fig 1). They were hyperintense on 3D-FLAIR and showed enhancement on 3D-T1weighted postcontrast imaging (Fig 2). They were not identifiable on routine 2D-T2WI TSE, T1WI, or DWI sequences, being isointense to CSF (Fig 3). They did not show a susceptibility effect. In 2 cases in which a heavily T2-weighted TSE sequence with a $90^{\circ}$ flip-back pulse was performed (T2 driven equilibrium radiofrequency reset pulse), the lesions were hypointense to CSF (Fig 3). In 9 cases, on 3D-T1-weighted postcontrast imaging, the lesions appeared continuous cranially and caudally, with a minute enhancing vascular structure passing from the posterior aspect of the vertebral artery dural penetration through the foramen magnum to the dura overlying the anterior condylar venous confluence (Fig 4). In all cases, this vascular structure was clearly separate from the posterior inferior cerebellar artery and did not represent a duplication of this artery or of the vertebral artery. Two patients underwent cerebral angiography subsequent to the MR imaging (basilar tip aneurysm and superior sagittal sinus dural arteriovenous fistula), and in both cases despite vertebral in- jections, no arterial or venous structure was identified to explain the enhancing lesion on MR imaging.

Nine patients and 10 lesions had longitudinal imaging follow-up between 6 and 77 months, in which all lesions remained stable in size and imaging characteristics. Details of patient demographics, scan indications and findings, and follow-up details are available in the On-line Table. Four patients had headaches as an indication for their MR imaging; none of these were occipital in nature. A potential cause for the headache was identified in only 1 patient who experienced vertex region headaches and had a superior sagittal sinus dural arteriovenous fistula with cortical venous reflux.

\section{DISCUSSION}

This cases series describes a foramen magnum finding on MR imaging that is almost certainly benign, but one that may simulate disease. The characteristics of this lesion are highly consistent, being a small 3D-FLAIR high-signal round or ovoid lesion posterior to the intradural vertebral artery that enhances on 3D-T1WI sequences. True pathology, which this lesion may mimic, includes meningioma, schwannoma, vertebral artery dissecting aneurysm, and meningeal or dural deposits from malignant or inflammatory disease. Such pathology has imaging characteristics differing from those of this benign lesion and should be discriminated mainly on the basis of location and T2WI appearances (this benign lesion was occult on 2D-TSE T2WI). Cystic lesions, which have also been reported in the foramen magnum, include arachnoid cysts, neuroenteric cysts, and synovial cysts. These cystic lesions may be differentiated by their location and being nonenhancing. 


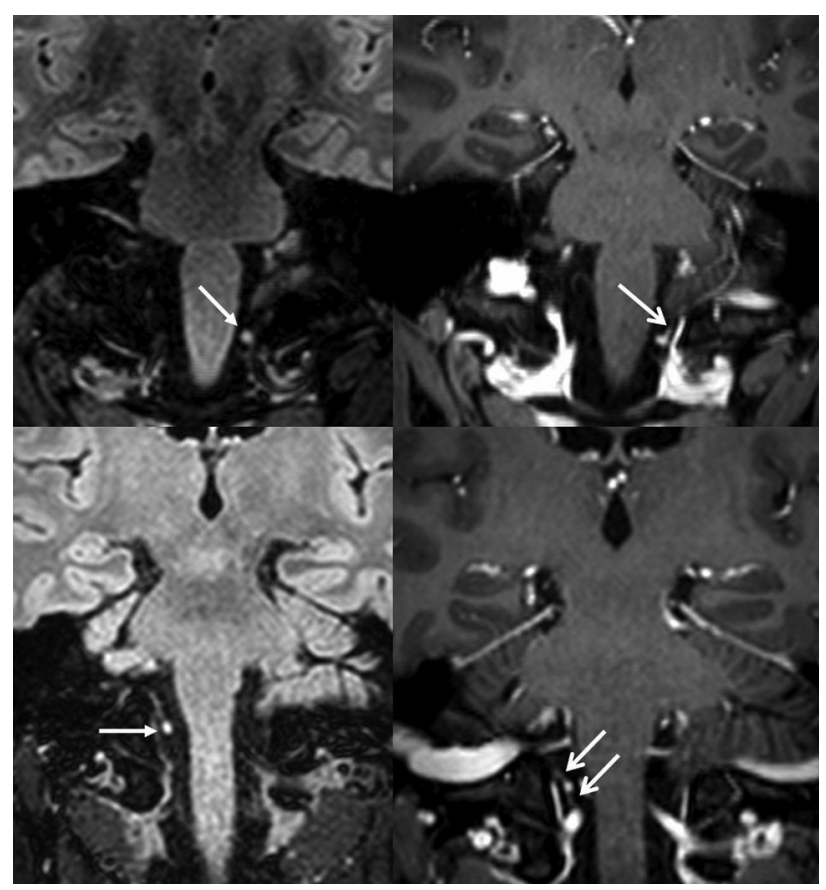

FIG 4. Two examples of a possible venous vascular connection with the lesion. Coronal FLAIR images (upper and lower left images) show typical hyperintense foci (white arrows). Corresponding coronal reformats of 3D-T1-weighted postcontrast images (upper and lower right images) show small enhancing linear channels (white arrows) connecting with the lesions.

In 9 cases, a direct and continuous connection was seen between the lesion and an intradural vein connecting the internal vertebral venous plexus near the dural penetration of the vertebral artery to the anterior condylar venous confluence. Such a connection was seen only in those patients who had high-quality 3D-T1weighted postcontrast scans without movement artifacts, potentially explaining why a connection was not seen in all cases. Therefore, this lesion may represent a small varix related to this vein. The vein is likely a bridging vein connecting the longitudinal venous channels on the surface of the lower medulla and upper cervical cord to the internal vertebral venous plexus. These bridging veins usually, but not invariably, traverse the dura with spinal nerve roots. ${ }^{3}$ More cranially at about the level of foramen magnum, further bridging veins are known to connect the inferior cerebellar peduncular veins with the venous sinuses converging on the jugular foramen. In addition a bridging vein may connect either the lateral anterior medullary or the lateral medullary vein with the marginal sinus or the veins of the hypoglossal canal. ${ }^{4,5}$

Other known anatomic structures that lie in relation to the posterior surface of the vertebral artery just beyond the dural penetration include the ventral rootlets of the $\mathrm{C} 1$ nerve, the posterior spinal artery, the upper triangular attachment of the dentate ligament, the dorsal rootlets of the $\mathrm{C} 1$ nerve, and the roots of the spinal accessory nerve (Fig 5). ${ }^{6}$ A ganglion or pseudoganglion lesion of either the $\mathrm{C} 1$ nerve or spinal roots of the spinal accessory nerve is a possible explanation for this lesion. A dorsal root ganglion of the $\mathrm{C} 1$ nerve has been identified in 30\% of sides during cadaveric dissection, with two-thirds of these ganglia lying medial to the intervertebral foramen. ${ }^{7}$ Macroscopic foci of enlargement

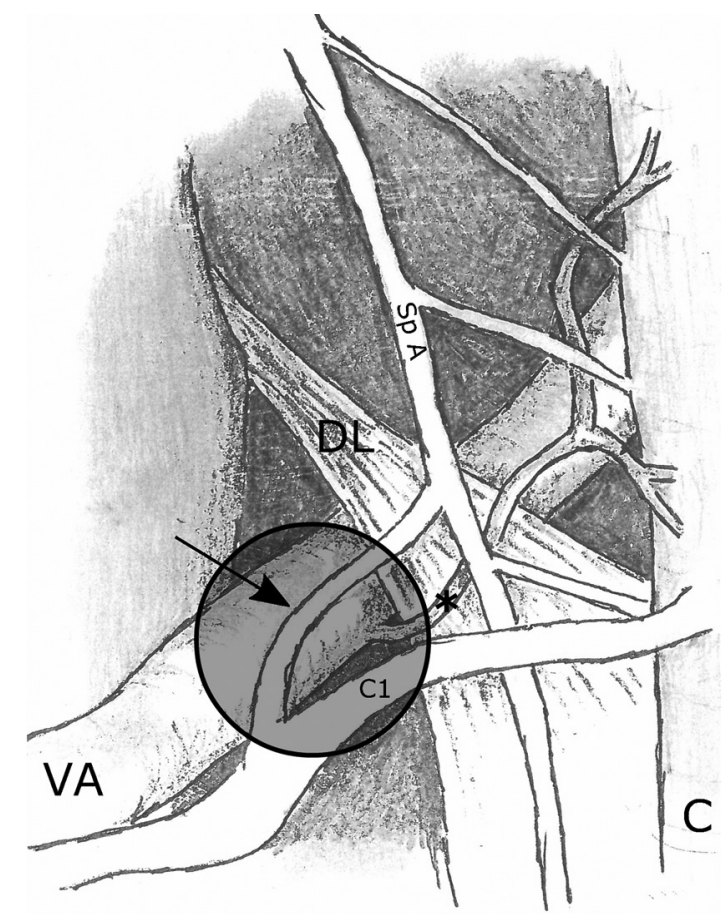

FIG 5. Diagram showing the posterior view of the left side of the intradural spinal canal at and just below the level of foramen magnum. The typical location of the foramen magnum lesion is shown by the shaded circle. Relationships of the vertebral artery (VA), dorsal root of the $\mathrm{Cl}$ nerve $(\mathrm{Cl})$, spinal accessory nerve (Sp A), dentate ligament (DL), posterior spinal artery (asterisk), and spinal cord (C) are shown. Note the typically present communicating branch (arrow) between the spinal accessory nerve and the $\mathrm{Cl}$ nerve.

of the spinal roots of the spinal accessory nerve, all occurring within $8.5 \mathrm{~mm}$ of the foramen magnum, have been identified and shown to represent ectopic glial rests or heterotopias rather than true ganglia. ${ }^{8}$ Nevertheless, neuronal cell bodies have been identified histologically along the spinal accessory nerve, which may have important considerations for neurally mediated occipital and other head and neck pain syndromes. ${ }^{9}$

The most likely reason for the recent identification of this lesion is the routine use of $3 \mathrm{D}$-FLAIR and, when contrast is required, 3D-T1 postcontrast sequences. 2D-FLAIR sequences frequently have CSF flow artifacts, which would obscure the lesion within the foramen magnum. ${ }^{10}$ The increased resolution and vascular flow signal return from 3D-T1 postcontrast sequences would allow the enhancement and identification of this potentially venous or neural structure. ${ }^{11}$ The lesion is likely to be occult on 2D-TSE T1-weighted postcontrast scans.

Limitations of this study include the lack of follow-up of 5 patients and 6 lesions. The primary reason was that these were the later patients in the series, by which time this entity was recognized by the reporting radiologists as a benign finding and either not reported or reported as not requiring any follow-up. Additionally, it would be ideal to have histologic proof of the benign nature of these lesions, but we believe this is unlikely to be obtained because they do not produce local mass effect and the risks of such an operation would outweigh any benefit. 


\section{CONCLUSIONS}

We describe a 3D-FLAIR hyperintense and enhancing lesion within the foramen magnum that is almost certainly a benign incidental finding (possibly a varix or a ganglion) but which may mimic other neoplastic and vascular pathology.

\section{REFERENCES}

1. Samii M, Gerganov VM. Surgery of extra-axial tumor of the cerebral base. Neurosurgery 2008;62:1153-66; discussion 1166-68 Medline

2. Talacchi A, Biroli A, Soda C, et al. Surgical management of ventral and ventrolateral foramen magnum meningiomas: report on a 64case series and review of the literature. Neurosurg Rev 2012;35:35967; discussion 367-68 CrossRef Medline

3. Krings T, Geibprasert S. Spinal dural arteriovenous fistulas. AJNR Am J Neuroradiol 2009;30:639-48 CrossRef Medline

4. Rhoton AL. The posterior fossa veins. Neurosurgery 2000;47:S69-92 CrossRef Medline

5. Hetts SW, English JD, Stiver SI, et al. Bilateral cervical spinal arteriovenous fistulas with intracranial venous drainage mimicking a foramen magnum dural arteriovenous fistula. Interv Neuroradiol 2013;19:483-88 Medline

6. Rhoton AL. The foramen magnum. Neurosurgery 2000;47:S155-93 CrossRef Medline

7. Tubbs RS, Loukas M, Yalçin B, et al. Classification and clinical anatomy of the first spinal nerve: surgical implications. J Neurosurg Spine 2009;10:390-94 CrossRef Medline

8. Tubbs RS, Lancaster JR, Mortazavi MM, et al. Do grossly identifiable ganglia lie along the spinal accessory nerve? A gross and histological study with potential neurosurgical significance. World Neurosurg 2012;77:349-51 CrossRef Medline

9. Tubbs RS, Sorenson EP, Watanabe K, et al. Histologic conformation of neuronal cell bodies along the spinal accessory nerve. $\mathrm{Br} \mathrm{J} \mathrm{Neu}$ rosurg 2014;28:746-49 CrossRef Medline

10. Kallmes DF, Hui FK, Mugler JP 3rd. Suppression of cerebrospinal fluid and blood flow artifacts in FLAIR MR imaging with a singleslab three-dimensional pulse sequence: initial experience. Radiology 2001;221:251-55 Medline

11. Pui MH, Fok ECM. MR imaging of the brain: comparison of gradient-echo and spin-echo pulse sequences. AJR Am J Roentgenol 1995; 165:959-62 CrossRef Medline 